\title{
The Mas Receptor as a Future Perspective in Parkinson's Disease
}

\author{
Mostafa A. Rabie, Mai A. Abd El Fattah, Noha N. Nassar, Dalaal M. Abdallah, Hanan S. El-Abhar* \\ Department of Pharmacology and Toxicology Faculty of Pharmacy, Cairo University, Cairo, Egypt
}

Article Info

\section{Article Notes}

Received: July 26, 2018

Accepted: August 07, 2018

\section{${ }^{*}$ Correspondence}

Dr. Hanan S. El-Abhar, Department of Pharmacology and Toxicology, Faculty of Pharmacy, Cairo University, 11562, Cairo, Egypt; Fax No: +202/24073411;

E-mail: hanan.elabhar@pharma.cu.edu.eg

(c) $2018 \mathrm{El}$-Abhar HS. This article is distributed under the terms of the Creative Commons Attribution 4.0 International License

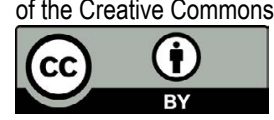

Abstract

The current review sheds light on the importance of the cerebral renin angiotensin system (RAS) in Parkinson's disease (PD), as a neurodegenerative disorder. Local RAS has been identified in the nigrostriatal pathway and plays a pivotal role in the progression of PD via binding to the upregulated angiotensin II type-1 receptor (AT-1R). Activation of AT-1R induces an oxidative stress neuroinflammatory status that results in further deterioration of dopaminergic neurons. Mas receptor (MasR) together with AT-2R, are considered major components of RAS protective arm, which mediates actions opposing AT-1R activation. Accordingly, angiotensin converting enzyme-2 (ACE-2)/Angiotensin 1-7 (Ang 1-7)/MasR axis has been emerged as a novel therapeutic target to ameliorate the devastating effects of Ang II/AT-1R cue in a 6-hydroxydopamine (OHDA) lesioned PD model. Stimulation of MasR via its physiological ligand Ang 1-7 attenuated 6-OHDA induced neurotoxicity as evidenced by the improved motor performance and coordination along with the preservation of the dopaminergic neurons. Via enhanced MasR/PI3K/Akt/ CREB/ BDNF/TrKB neurogenesis cascade, besides both antioxidants and anti-inflammation mechanisms, Ang 1-7 proved its therapeutic benefits in PD, partly through MasR, to open up a new avenue for treatment.

\section{Introduction}

Parkinson's disease (PD) is the second leading most common neurodegenerative disease after Alzheimer's disease (AD) that is currently afflicting more than $1 \%$ of those older than 60 years and up to $4 \%$ of those older than 80 years ${ }^{1}$. PD is characterized by a progressive loss of the dopaminergic neurons in the substantia nigra pars compacta (SNpc) with the presence of intra-neuronal proteinaceous cytoplasmic inclusions of Lewy bodies. This results in a marked deficiency in striatal dopamine (DA) and the appearance of major clinical symptoms of PD namely; akinesia, muscle rigidity and resting tremor ${ }^{2,3}$. Currently, no cure or disease-modifying therapy that stops or slows disease progression has been proven in PD. Treatments for PD are not always effective in the early stage of PD, indicating that other approaches are needed to be uncovered to halt the progressive loss of SN dopaminergic neurons. Eventually, preserving viable nigrostriatal neurons could delay disease progression and prolong the efficacy of treatments ${ }^{4}$.

Neurotrophic factors (NTFs) have shown effectiveness in neurodegenerative disorders including PD preclinical studies; however, their exact role to manage PD motor symptoms is still elusive. The goal of this mini-review is to gather the currently available data and to address the potential future prospects for Mas receptor (MasR)/ brain-derived neurotrophic factor (BDNF) activation as an innovative approach to manage PD. 


\section{Etiopathology of PD: role of BDNF}

Several genes such as; alpha-synuclein ( $\alpha$-syn) and parkin are deleted or mutated of in the early onset familial $\mathrm{PD}$, while in late-onset sporadic idiopathic PD, which accounts for most PD cases, environmental factors play the major role. Though the clinical phenotype of PD is relatively homogeneous, the pathogenic mechanism seems to be multifactorial. A constellation of events has been implicated in the degeneration of dopaminergic neurons in $\mathrm{PD}$, including mitochondrial dysfunction, microglia activation, neuroinflammation, an enhanced oxidative milieu, accumulation of aberrant or misfolded proteins, and ubiquitin-proteasome system dysfunction ${ }^{5,6}$.

A multitude of studies implicated an imperative role for BDNF and cAMP response element binding (CREB), among other protective factors, in regulating synaptic plasticity in PD. Indeed, BDNF supports differentiation, maturation, and survival of neurons in the $\mathrm{CNS}^{7}$. This trophic factor is co-localized with SN dopaminergic neurons to function as a neuronal preservative molecule in PD. Indeed, BDNF inhibition participates in the death of nigral dopaminergic neurons ${ }^{8}$, since BDNF acts as a compensatory mechanism to halt the disease progression ${ }^{9}$. Actually, a $70 \%$ reduction in SNpc BDNF mRNA expression has been reported in both clinical ${ }^{10}$ and neuropathological typical $\mathrm{PD}^{11}$. Recently in the pathogenesis of PD, $\alpha$-syn-induces axonal dysfunction by impairing trafficking and signaling of BDNF as shown in a mouse PD model ${ }^{12}$. The DATATOP trial revealed a slower PD symptoms progression in early unmedicated PD subjects with the minor/minor rs6265 genetic BNDF variant that delayed the need for dopaminergic medications ${ }^{13}$.

The partial deletion of the BDNF receptor tyrosine kinase B (TrKB), with the consequent suppressed BDNF biosynthesis, reduces SN neuronal count and tyrosine hydroxylase (TH) expression associated by $\alpha$-syn deposition $^{14}$. On the other side, dysfunctional mutated parkin overexpression prevents dopaminergic neuronal regeneration via the deactivation of the CREB pathway to contribute to $\mathrm{PD}^{15}$.

\section{Role of the brain local RAS in dopaminergic neuronal degeneration}

In addition to the humoral classical and the intracrine renin-angiotensin system (RAS), a local RAS has been recognized in the basal ganglia ${ }^{16}$; the understanding of these systems can establish new perspectives for the management of RAS-related diseases, such as $\mathrm{PD}^{17}$. The local RAS in the basal ganglia includes high concentrations of angiotensin converting enzyme (ACE), angiotensin II (Ang II), angiotensin II type-1 receptor (AT-1R), AT$2 \mathrm{R}$, prorenin receptors, and NADPH oxidase enzyme (Nox) ${ }^{18}$. Ang II is the main effector peptide of the RAS that acts via AT- $1 \mathrm{R}$ and $\mathrm{AT}-2 \mathrm{R}^{19}$. Beside a cohort study in hypertensive patients that suggested a protective effect of the ACE-inhibitors (ACE-Is) for PD risk ${ }^{20}$, a plethora of experimental data supports the involvement of brain RAS in dopaminergic neuronal degeneration in the $\mathrm{SN}^{21,22}$. The abnormal upregulation of local Ang II exacerbates neuro-inflammation and induces oxidative stress ${ }^{23}$, hence, constituting early components of dopaminergic cell death by acting synergistically with other factors to progress $\mathrm{PD}^{24,25}$. Importantly, Nox inhibitors [26], as well as ACEIs ${ }^{22,27}$ and AT-1R blockade ${ }^{26,28}$ were reported to hinder neuronal loss in PD models. Of note, depletion of DA with reserpine or 6- hydroxydopamine (OHDA) provoked chronic dopaminergic denervation and increased the expression of AT-1R, besides the Nox-complex activity. These events were decreased as the DA function was restored ${ }^{29}$ or upon the administration of levodopa (L-DOPA ${ }^{30} ;$ ).

\section{ACE-2/Ang 1-7/MasR signaling cascade}

In the brain, Ang 1-7 is primarily formed via the degradation of Ang II by ACE-2 to activate the MasR that is widely distributed in the cerebrum. It was located in the cerebral cortex, basal ganglia ${ }^{31}$, in dopaminergic neurons, and glial cells in rat $\mathrm{SNpc}$, as well as mesencephalic primary cultures $^{32}$. The effects mediated by Ang 1-7 are in direct opposition to the actions of Ang II/AT-1R axis. Indeed, MasR can hetero-oligomerize with AT-1R to act as a direct antagonist ${ }^{33}$ in the neurons ${ }^{34}$ and microglia ${ }^{35}$. Moreover, Ang 1-7-mediated NF- $\mathrm{KB}$ inhibition has been also documented in several animal models, including hypertensive kidney disease $^{36}$, pulmonary fibrosis ${ }^{37}$, and fatty liver disease ${ }^{38}$.

The neuroprotective effect of Ang 1-7 was signified earlier in an ischemic stroke mode ${ }^{39}$ and in 2011, Mecca et $\mathrm{al}^{40}$, found that the intracerebroventricular infusion of Ang 1-7 attenuated the cerebral infarct size and neurological deficits elicited by endothelin-1-induced middle cerebral artery occlusion. Moreover, Ang 1-7 prevented Shiga toxin type 2-induced neuronal apoptosis, glial ultrastructural alterations, and demyelinated fibers in the rat corpus striatum ${ }^{41}$. Additionally, activation of ACE-2/Ang 1-7/ MasR axis opposed AT-1R mediated apoptosis and reduced cognitive deficits in an $\mathrm{AD}^{42}$. Recently, Ang 1-7 inhibited the increase in cellular reactive oxygen species (ROS) produced by Ang II in dopaminergic neurons in vitro, confirming, thus, a neuroprotective role of the Ang 1-7/MasR axis ${ }^{32}$.

\section{Role of the Activation of the Ang-1-7/Masr Cue as a Therapy for PD}

Local bone marrow (BM) RAS regulates hematopoiesis, erythropoiesis, and myelopoiesis, in addition to formation of monocytic and lymphocytic lineages. The local RAS affects tumor growth and metastases via the modulation of various carcinogenic events, including angiogenesis, apoptosis, cellular proliferation, immune responses, as well as cell signaling and extracellular matrix formation 
in an autocrine and paracrine manner. Targeting local RAS actions could be a promising therapeutic potential for the management of neoplastic disorders ${ }^{43}$.

In our study, a novel role for MasR activation by Ang 1-7 in PD has been established to open up a new avenue for the treatment of this neurodegenerative disease ${ }^{44}$. The unilateral intrastriatal Ang 1-7 post-administration improved motor performance and muscle coordination in a 6-OHDA PD model. Ang 1-7 was able to upregulate the striatal expression of MasR and stimulate the BDNF/TrKB axis. This was mediated by the activation of phosphoinositide 3-kinase /protein kinase B /CREB (PI3K/Akt/CREB) trajectory. Moreover, the MasR ligand Ang 1-7 preserved dopaminergic neurons by increasing SN TH immunoreactivity to enhance DA. Ang 1-7 also downregulated the striatal expression of AT-1R to halt neurodegeneration as indicated by the profound reduction in MAPK p38/NF- $\kappa B$ p65, as well as Nox/lipid peroxidation. Notably, both MasR cue activation and AT-1R cascade repression were in part reversed by the selective MasR antagonist A-779.

\section{Conclusion}

According to the previous data, we can conclude that activation of the MasR instigates survival dopaminergic neuronal signals and abrogates local RAS in the nigrostriatal pathway to advocate it as a potential therapeutic target for the management of PD.

\section{References}

1. de Lau LM, Breteler MM. Epidemiology of Parkinson's disease. Lancet Neurol. 2006; 5(6): 525-535.

2. Bergman H, Deuschl G. Pathophysiology of Parkinson's disease: from clinical neurology to basic neuroscience and back. Mov Disord. 2002; 17 Suppl 3: S28-S40.

3. Blesa J, Trigo-Damas I, Quiroga-Varela A, et al. Oxidative stress and Parkinson's disease. Front Neuroanat. 2015; 9: 91.

4. Kordower JH, Embor M.E, Bloch J, et al. Neurodegeneration prevented by lentiviral vector delivery of GDNF in primate models of Parkinson's disease. Science. 2000; 290(5492): 767-773.

5. Jenner P, Olanow CW. The pathogenesis of cell death in Parkinson's disease. Neurology. 2006; 66(10 Suppl 4): S24-S36.

6. Olanow CW. The pathogenesis of cell death in Parkinson's disease 2007. Mov Disord. 2007; 22 Suppl 17: S335-S342.

7. Huang EJ, Reichardt LF. Neurotrophins: roles in neuronal development and function. Annu. Rev. Neurosci. 2001; 24: 677-736.

8. Porritt MJ, Batchelor PE, Howells DW. Inhibiting BDNF expression by antisense oligonucleotide infusion causes loss of nigral dopaminergic neurons. Exp Neurol. 2005; 192(1): 226-234.

9. Scalzo P, Kummer A, Bretas TL, et al. Serum levels of brain-derived neurotrophic factor correlate with motor impairment in Parkinson's disease. J. Neurol. 2010; 257(4): 540-545.

10. Howells DW1, Porritt MJ, Wong JY, et al. Reduced BDNF mRNA expression in the Parkinson's disease substantia nigra. Exp Neurol. 2000; 166(1): 127-135.
11. Shen T, You Y, Joseph C, et al. BDNF Polymorphism: A Review of Its Diagnostic and Clinical Relevance in Neurodegenerative Disorders. Aging Dis. 2018; 9(3): 523-536

12. Fang F, Yang W, Florio JB, et al. Synuclein impairs trafficking and signaling of BDNF in a mouse model of Parkinson's disease. Sci Rep. 2017; 7(1): 3868.

13. Fischer DL, Auinger P, Goudreau JL, et al. Bdnf variant is associated with milder motor symptom severity in early-stage Parkinson's disease. Parkinsonism Relat Disord. 2018.

14. von Bohlen und Halbach O, Minichiello L, Unsicker $\mathrm{K}$. Haploinsufficiency for trkB and trkC receptors induces cell loss and accumulation of alpha-synuclein in the substantia nigra. FASEB J. 2005; 19(12): 1740-1742.

15. Ham S, Lee YI, Jo M, et al. Hydrocortisone-induced parkin prevents dopaminergic cell death via CREB pathway in Parkinson's disease model. Sci Rep. 2017; 7(1): 525.

16. Allen AM, MacGregor DP, Chai SY, et al. Angiotensin II receptor binding associated with nigrostriatal dopaminergic neurons in human basal ganglia. Ann Neurol. 1992; 32(3): 339-344.

17. Labandeira-García JL, Garrido-Gil P, Rodriguez-Pallares J, et al. Brain renin-angiotensin system and dopaminergic cell vulnerability. Front Neuroanat. 2014; 8: 67.

18. Labandeira-Garcia JL, Rodriguez-Pallares J, Rodríguez-Perez AI, et al. Brain angiotensin and dopaminergic degeneration: relevance to Parkinson's disease. Am J Neurodegener Dis. 2012; 1(3): 226-244.

19. Oro C, Qian H, Thomas WG. Type 1 angiotensin receptor pharmacology: signaling beyond G proteins. Pharmacol Ther. 2007; 113(1): 210-226.

20. Perez-Lloret S, Otero-Losada M, Toblli JE, et al. Renin-angiotensin system as a potential target for new therapeutic approaches in Parkinson's disease. Expert Opin Investig Drugs. 2017; 26(10): 11631173.

21. Konings CH, Kuiper MA, Bergmans PL, et al. Increased angiotensinconverting enzyme activity in cerebrospinal fluid of treated patients with Parkinson's disease. Clin Chim Acta. 1994; 231(1): 101-106.

22. Sonsalla PK, Coleman C, Wong LY, et al. The angiotensin converting enzyme inhibitor captopril protects nigrostriatal dopamine neurons in animal models of parkinsonism. Exp Neurol. 2013; 250: 376-383.

23. Wright JW, Harding JW. The brain renin-angiotensin system: a diversity of functions and implications for CNS diseases. Pflugers Arch. 2013; 465(1): 133-151.

24. Wu DC, Teismann P, Tieu K, et al. NADPH oxidase mediates oxidative stress in the 1-methyl-4-phenyl-1,2,3,6-tetrahydropyridine model of Parkinson's disease. Proc Natl Acad Sci U S A. 2003; 100(10): 61456150.

25. Rodriguez-Pallares J, Parga JA, Muñoz A, et al. Mechanism of 6-hydroxydopamine neurotoxicity: the role of NADPH oxidase and microglial activation in 6-hydroxydopamine-induced degeneration of dopaminergic neurons. J Neurochem. 2007; 103(1): 145-156.

26. Rodriguez-Pallares J, Rey P, Parga JA, et al. Brain angiotensin enhances dopaminergic cell death via microglial activation and NADPH-derived ROS. Neurobiol Dis. 2008; 31(1): 58-73.

27. Lopez-Real A, Rey P, Soto-Otero R, et al. Angiotensin-converting enzyme inhibition reduces oxidative stress and protects dopaminergic neurons in a 6-hydroxydopamine rat model of Parkinsonism. $J$ Neurosci Res. 2005; 81(6): 865-873.

28. Sánchez-Iglesias S, Rey P, Méndez-Alvarez E, et al. Time-course of brain oxidative damage caused by intrastriatal administration of 6-hydroxydopamine in a rat model of Parkinson's disease. Neurochem Res. 2007; 32(1): 99-105. 
29. Labandeira-Garcia JL, Rodriguez-Pallares J, Villar-Cheda B, et al. Aging Angiotensin system and dopaminergic degeneration in the substantia nigra. Aging Dis. 2011; 2(3): 257-274.

30. Villar-Cheda B, Rodríguez-Pallares J, Valenzuela R, et al. Nigral and striatal regulation of angiotensin receptor expression by dopamine and angiotensin in rodents: implications for progression of Parkinson's disease. Eur J Neurosci. 2010; 32(10): 1695-1706.

31. Becker LK, Etelvino GM, Walther T, et al. Immunofluorescence localization of the receptor Mas in cardiovascular-related areas of the rat brain. Am J Physiol Heart Circ Physiol. 2007; 293(3): H1416-H1424.

32. Costa-Besada MA, Valenzuela R, Garrido-Gil P, et al. Paracrine and Intracrine Angiotensin 1-7/Mas Receptor Axis in the Substantia Nigra of Rodents, Monkeys, and Humans. Mol. Neurobiol. 2017.

33. Kostenis E, Milligan G, Christopoulos A, et al. G-protein-coupled receptor Mas is a physiological antagonist of the angiotensin II type 1 receptor. Circulation. 2005; 111(14): 1806-1813.

34. Deliu E, Brailoiu GC, Eguchi S, et al. Direct evidence of intracrine angiotensin II signaling in neurons. Am J Physiol Cell Physiol. 2014; 306(8): C736-C744.

35. Regenhardt RW, Desland F, Mecca AP, et al. Anti-inflammatory effects of angiotensin-(1-7) in ischemic stroke. Neuropharmacology. 2013 71: 154-163.

36. Giani JF, Muñoz MC, Pons RA, et al. Angiotensin-(1-7) reduces proteinuria and diminishes structural damage in renal tissue of stroke-prone spontaneously hypertensive rats. Am J Physiol Renal Physiol. 2011; 300(1): F272-F282.

37. Meng $\mathrm{Y}, \mathrm{Yu} \mathrm{CH}, \mathrm{Li}$ W, et al. Angiotensin-converting enzyme 2/
angiotensin-(1-7)/Mas axis protects against lung fibrosis by inhibiting the MAPK/NF-kappaB pathway. Am J Respir Cell Mol Biol. 2014; 50(4): 723-736.

38. Santos RA, Ferreira AJ, Verano-Braga T, et al. Angiotensin-converting enzyme 2, angiotensin-(1-7) and Mas: new players of the reninangiotensin system. J Endocrinol. 2013; 216(2): R1-R17.

39. Bennion DM, Haltigan E, Regenhardt RW, et al. Neuroprotective mechanisms of the ACE2-angiotensin-(1-7)-Mas axis in stroke. Curr Hypertens Rep. 2015; 17(2): 3.

40. Mecca AP, Regenhardt RW, O'Connor TE, et al. Cerebroprotection by angiotensin-(1-7) in endothelin-1-induced ischaemic stroke. Exp Physiol. 2011; 96(10): 1084-1096.

41. Goldstein J, Loidl CF, Creydt VP, et al. Intracerebroventricular administration of Shiga toxin type 2 induces striatal neuronal death and glial alterations: an ultrastructural study. Brain Res. 2007; 1161: 106-115.

42. Uekawa K, Hasegawa Y, Senju S, et al. Intracerebroventricular Infusion of Angiotensin-(1-7) Ameliorates Cognitive Impairment and Memory Dysfunction in a Mouse Model of Alzheimer's Disease. J Alzheimers Dis. 2016; 53(1): 127-133.

43. Haznedaroglu IC, Beyazit Y. Local bone marrow renin-angiotensin system in primitive, definitive and neoplastic haematopoiesis. Clin Sci (Lond). 2013; 124(5): 307-323.

44. Rabie MA, Abd El Fattah MA, Nassar NN, et al. Angiotensin 1-7 ameliorates 6-hydroxydopamine lesions in hemiparkinsonian rats through activation of MAS receptor/PI3K/Akt/BDNF pathway and inhibition of angiotensin II type-1 receptor/NF-kappaB axis. Biochem Pharmacol. 2018; 151: 126-134. 